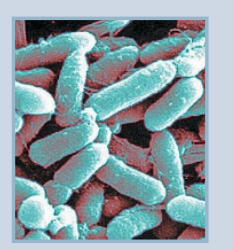

p1229 Bugs as drugs: Bacteria may be the next big thing in cancer treatment.

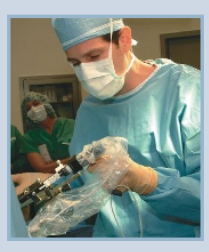

p1230 Brain twisters: Why does Parkinson disease attract unconventional therapies?

\title{
Marijuana researchers reach for pot of gold
}

The words 'medical marijuana' instantly conjure images of agitated politicians and emotional patients, each heatedly arguing their point of view. Thanks to marijuana's popularity as a recreational drug, the debates are usually informed more by moral and cultural beliefs than by rigorous scientific evidence.

But the real story is quietly unfolding behind the politics, where cannabis research has been seeping into mainstream neuroscience. Since the discovery of the first endocannabinoidthe body's own chemical that resembles those found in cannabis - in 1992, scientists have been busy unraveling what appears to be a major neurotransmitter system with an astounding panoply of effects.

What they've found so far suggests that the endocannabinoid system has an important role in nearly every paradigm of pain, in memory, in neurodegeneration and in inflammation. "There is no major biological system where these compounds are not involved," says Israeli researcher Raphael Mechoulam, who is credited with several seminal findings in the field.

If cannabinoids are crucial to so many biological processes, it follows that their clinical potential is enormous. Until recently, however, most of the evidence for cannabinoid use had been anecdotal because many countries are reluctant to fund research on its medical potential.

Marijuana has been banned in the US since 1937 and in the UK since 1971. A few countries support its medical use: Mechoulam gets his 'hash' from the Israeli police, Canada legalized medical marijuana in 2001, and the Netherlands sanctioned its use in September.

Cannabis sativa contains more than 60 active compounds, the most active of which is delta-9tetrahydrocannabinol (THC). People have used cannabis to treat all manner of pain, nausea, muscle cramps, glaucoma, asthma, strokes and insomnia. A few small clinical trials have confirmed its efficacy in managing some of these.

Critics point to marijuana's ability to induce anxiety, panic and paranoia. But a recent metaanalysis concluded that there is no substantial effect of its long-term use on the brain. "Compared to many of the drugs doctors prescribe to their patients, marijuana is a pretty harmless substance," says Donald Abrams, pro- fessor of medicine at the University of California in San Francisco (UCSF).

Abrams' experience with clinical trials illustrates how inextricably marijuana research is linked with politics. Abrams first applied to the National Institute on Drug Abuse, the only legal source of marijuana in the US, for funds to study marijuana's effects on AIDS patients. After twice being rejected, he reworded his proposal to highlight the drug's potential negative effects. He received nearly $\$ 1$ million in funding.

The US government has not initiated a study evaluating medical marijuana in two decades. Because marijuana is classified as a schedule I drug — with a high potential for abuse and no medical use-cannabis research faces scrutiny by four federal agencies.

"I can go out and get cannabis on the street easier than [I can get] candy," says Roger Nicoll, a pharmacologist at UCSF. "There is a total disconnect between the use of marijuana and the way in which the government is controlling it."

Most researchers say the scales may tip in marijuana's favor if researchers can find a way to deliver therapy free of its 'high'. The key may lie in basic research on the cannabinoid receptors.

There are two known cannabinoid receptors, $\mathrm{CB} 1$ and $\mathrm{CB} 2$. CB1 is the most abundant Gprotein-coupled receptor in the brain, and its distribution there hints at intriguing functions: it is ubiquitous in the cerebellum, but in the hippocampus - the memory center-it is only found in a subclass of neurons that regulate memory storage and retrieval.

$\mathrm{CB} 2$, in contrast, is found only in peripheral sites with immune functions. Some researchers suggest that using $\mathrm{CB} 2$-specific compounds may be one way to avoid psychotropic effects. Mechoulam, at Hebrew University in Jerusalem, has developed one such compound unevocatively called HU308, and a US team has reported preliminary results with another molecule.

Mechoulam is also focusing on cannabidiol, the second biggest ingredient in cannabis and the least psychoactive. Cannabidiol is known to be a potent agent against rheumatoid arthritis. Based on a synthetic cannabidiol developed by Mechoulam, the Israeli company Pharmos is conducting clinical trials of the compound's effects on brain injury. UK-based GW

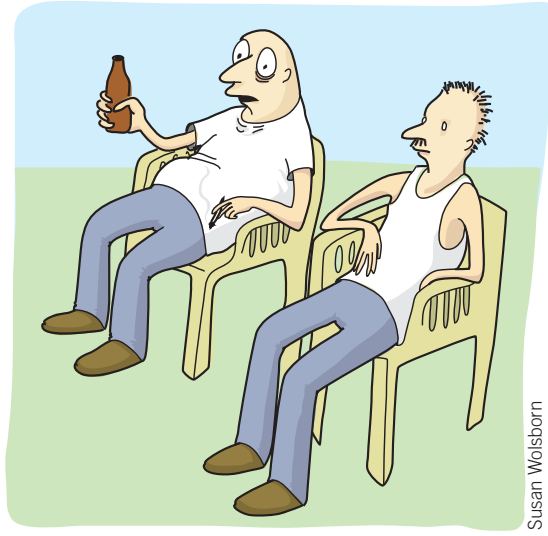

The marijuana is great for my glaucoma, but now I'm drinking a lot more beer to ease the paranoia.

Pharmaceuticals is testing cannabis extracts with different ratios of THC and cannabidiol.

Cannabidiol's mechanism of action is unknown, in part because it does not bind to the known receptors. But Mechoulam suggests there may be more receptors-as well as endocannabinoids - to be found, opening up possibilities for specific manipulation of the system.

Delivery technologies for cannabis are another avenue of research. Many patients prefer to smoke cannabis because it takes effect quickly and they can titrate the doses better. But some scientists are uncomfortable with the idea of a smoked therapeutic. "There isn't a single drug that is being smoked-and for good reasons," says Mechoulam.

GW has developed a cannabis extract in a mouth spray. It is also working on nasal inhalers and tablets that dissolve under the tongue.

As evidence of marijuana's potential mounts and scientists discover ways to minimize its downside, governments are reconsidering their stance. A US National Institutes of Health report in 1997 and a report from the Institute of Medicine in 1990 both recommended more research to evaluate marijuana's medical potential. Based on the reports, California funded the first Center for Medicinal Cannabis Research.

Abrams, one of the researchers at the center, now runs three clinical trials. "The science is fascinating enough to keep my attention," Abrams says. "I'll leave the politics to other people."

Apoorva Mandavilli, New York 